\title{
Pricing Double Barrier Options by Combinatorial Approaches
}

\author{
Tian-Shyr Dai ${ }^{1}$ and Yuh-Dauh Lyuu ${ }^{2}$
}

1 Corresponding author. Department of Applied Mathematics, Chung-Yuan Christian University, $22 \mathrm{Pu}-J e n, \mathrm{Pu}-\mathrm{chung} \mathrm{Li}$, Chung-Li, Tao-Yuan county, Taiwan 320. E-mail: cameldai@cycu.edu.tw.

2 Department of Finance and Department of Computer Science \& Information Engineering (preferred address), National Taiwan University, No. 1, Sec. 4, Roosevelt Rd., Taipei, Taiwan. E-mail: lyuu@.csie.ntu.edu.tw. The author was supported in part by NSC grant 92-2213-E-002-016.

Summary. Double barrier options are important path-dependent derivatives in the financial market. How to price them efficiently and accurately is thus important. Until now, no simple closed-form pricing formula for double barrier options is reported. Double barrier options can be priced on a lattice that divides a certain time interval (from option initial date to maturity date) into $n$ equal-length time steps. The pricing results obtained by the lattice algorithm converge to the true option value as $n \rightarrow \infty$, and the results oscillate significantly especially when $n$ is not large enough. To obtain an accurate pricing result without suffering from price oscillation, $n$ is required to be a large number. Unfortunately, the lattice pricing algorithm runs in $O\left(n^{2}\right)$ time. This paper proposes a linear-time combinatorial algorithm that can generate the same pricing results as the lattice algorithm. Thus our algorithm can handle very large $n$ 's efficiently. This algorithm uses a novel technique based on the re ection principle and the inclusion-exclusion principle. Numerical experiments are given to verify the excellent performance of our algorithm.

\section{Introduction}

Path-dependent derivatives are financial derivatives whose payoff depends on the price history of the underlying asset, which is assumed to be stock for convenience. They are essential to speculation and the management of financial risk. An option is a financial derivative that gives their buyers the right to buy or sell the stock for a contractual price (the exercise price) at some certain date (the maturity date). Double barrier options are popular options in the financial market. How to price them efficiently and accurately is thus important.

A double barrier option is an option with two barriers (two certain predetermined price levels). The option begins to exist once the stock price has 


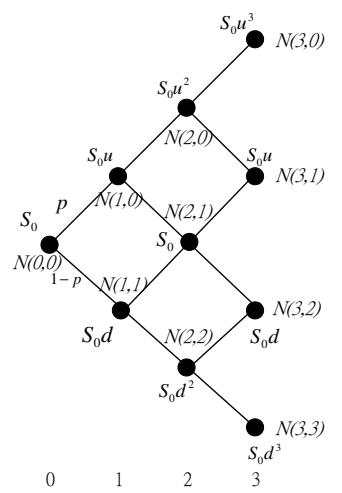

Fig. 1. The CRR Lattice. The initial stock p ice is $S_{0}$. The upwa d and downwa d multiplicative facto $\mathrm{s}$ fo the stock $\mathrm{p}$ ice a e and , espectively. The upwa $\mathrm{d}$ and downwa $\mathrm{d} b$ anching $\mathrm{p}$ obabilities a e and $1-$, espectively.

$\mathrm{r}$ ach $\mathrm{d}$ on of th barri rs. Th $\mathrm{r}$ ar no simpl clos d-form formulas to pric th doubl barri $\mathrm{r}$ option. Th $\mathrm{d} \mathrm{v}$ lopm nt of ffici nt num rical algorithms is thus critical.

Th valu of a doubl barri $r$ option can $b$ approximat $d \mathrm{~b}$ a lattic pricing algorithm. A lattic divid $\mathrm{s}$ th tim hori on of an option into discr t tim st ps. Tak a 3-tim -st p CRR lattic [2] in Fig. 1 as an xampl . (Th d tails of th CRR lattic will b d scrib d lat r.) Th tim int rval $\mathrm{b}$ tw $\mathrm{n}$ th option initial dat to th maturit dat is $\mathrm{v} \mathrm{nl}$ divid d into 3 tim st ps. Th stock pric at tim st $\mathrm{p} 0$ is $S_{0}$. Th stock pric can ith $\mathrm{r}$ mov up to $S_{0}$ or down to $S_{0} d$ at th first tim st p, wh $\mathrm{r} u$ and $d \mathrm{~d}$ not som constants. Similarl, ach stock pric can ith $\mathrm{r}$ mov up or mov down again in th following tim st ps. A traditional lattic pricing algorithm $\mathrm{n}$ ds to valuat th option valu at ach nod in th lattic. Sinc th $\mathrm{r}$ ar $O(n)$ nod s in a $n$-tim -st p lattic, th algorithm runs in tim $O(n)$. Th pricing $\mathrm{r}$ sults comput $\mathrm{d} b$ th CRR lattic conv rg to th tru option valu as $n \rightarrow \infty$. Mor ov $\mathrm{r}$, th pricing $\mathrm{r}$ sults oscillat significantl sp ciall wh $n$th numb $r$ of tim st ps $n$ of th lattic is small. An ffici nt num rical pricing algorithm that can handl larg $n$ is thus important. Ritchk $n[6]$ and Figl wski and Gao [3] tr to $r$ mov th pric oscillation $b$ modif ing th structur of th lattic, but th ir algorithms still run in tim $O(n)$. Oth $\mathrm{r}$ num rical approach $\mathrm{s}$ ar studi $\mathrm{d}$ in $[1,4,7]$. In this pap $\mathrm{r}$, w will provid a lin ar-tim combinatorial algorithm that produc $\mathrm{s}$ th sam pricing $\mathrm{r}$ sults as th lattic algorithm. Our combinatorial algorithm is th most ffici nt curr ntl known in th lit ratur.

Th dramatic improv $\mathrm{m}$ nt in this algorithm is don $\mathrm{b}$ using combinatorial formulas to simplif th calculation in th lattic pricing algorithm. Th combinatorial approach to $d$ rivativ s pricing is first introduc $d \mathrm{~b}$ [5]. This $\mathrm{m}$ thod $\mathrm{r}$ sults in th most ffici nt pricing algorithms for vanilla options and 
single barrier options. This paper uses two combinatorial tools, the reflection principle and the inclusion-exclusion principle, to design a linear-time algorithm for pricing double barrier options. Computer experiments will show the extreme efficiency of this algorithm.

This paper is organized as follows. The definition of the double barrier option is described in Section 2. How to price an option on a lattice is described in the same section. A linear-time pricing algorithm constructed by using the reflection principle and the inclusion-exclusion principle is introduced in Section 3. Numerical results given in Section 4 verifies the excellence performance of our algorithm. Section 5 concludes our paper.

\section{Preliminaries}

The options is assumed to initiate at time 0 and mature at time $T$. Let $S(t)$ denote the stock price at time $t$, where $0 \leq t \leq T$, and $X$ denote the exercise price, which is defined on the option contract.

\section{Vanilla Options}

A vanilla option gives the holder the right to buy or sell the underlying asset for the exercise price. The payoff of a vanilla option at time $T$ can be expressed as follows:

$$
\max (\theta S(T)-\theta X, 0),
$$

where $\theta$ is equal to 1 for call options and -1 for put options. The vanilla options can be priced by a linear-time combinatorial algorithm, which will be described later. We will also show that double barrier options can be priced by this linear-time algorithm under some degenerate cases.

\section{Double Barrier Options}

A double barrier option is an option whose payoff depends on whether the stock price reaches two predefined price levels $L$ and $H$ during the life of the option. Assume $L<H$ for convenience. Define $S_{\text {sup }}=\sup _{0 \leq t \leq T} S(t)$ and $S_{\text {inf }}=\inf _{0 \leq t \leq T} S(t)$. The payoff for a double barrier option can be expressed as follows:

$$
\text { Payoff }= \begin{cases}\max (\theta S(T)-\theta X, 0) & \text { if } S_{\mathrm{sup}} \geq H \text { or } S_{\mathrm{inf}} \leq L, \\ 0 & \text { otherwise }\end{cases}
$$

This paper focuses on the pricing of double barrier calls. The extension to double barrier puts is straightforward. 


\section{The CRR Lattice}

The standard CRR lattice [2] will be adopted for the stock price dynamics. A simple 3-time-step CRR lattice is illustrated in Fig. 1. Let $S_{i}$ denote the stock price at time step $i$. The CRR lattice says that $S_{i+1}$ equals $S_{i} u$ (the up move) with probability $p$ and $S_{i} d$ (the down move) with probability $1-p$, where $d<u$ and $u d=1$. The price $S_{i}$ resulting from $j$ down moves and $i-j$ up moves equals $S_{0} u^{i-j} d^{j}$ with probability $\left(\begin{array}{l}i \\ j\end{array}\right) p^{i-j}(1-p)^{j}$. This node (with corresponding stock price $S_{0} u^{i-j} d^{j}$ at time step $i$ ) is denoted as $N(i, j)$ for simplicity. Suppose that the CRR lattice starts at time step 0 and ends at time step $n$. For pricing purposes, $u$ is set as $e^{\sigma \sqrt{T / n}}$, where $\sigma$ denotes the volatility of the stock price. The probability $p$ is set to $\left(e^{r}-d\right) /(u-d)$, where $r$ denotes the continuously compounded risk-free interest rate per time step.

\section{Option Pricing on the CRR Lattice}

The value of an option is equal to the discounted expected payoff of this option at the maturity date. Take a vanilla call as an example. The payoff for a vanilla option at node $N(n, j)$ is $\max \left(S_{0} u^{n-j} d^{j}-X, 0\right)$. Since the probability to reach node $N(n, j)$ is $\left(\begin{array}{c}n \\ j\end{array}\right) p^{n-j}(1-p)^{j}$, so the value contributed by node $N(n, j)$ is $\left(\begin{array}{c}n \\ j\end{array}\right) p^{n-j}(1-p)^{j} \max \left(S_{0} u^{n-j} d^{j}-X, 0\right)$. Thus the value priced by a $n$-time-step lattice is equal to

$$
e^{-r n} \mathrm{E}(\text { Payoff })=e^{-r n} \sum_{j=0}^{n}\left(\begin{array}{l}
n \\
j
\end{array}\right) p^{n-j}(1-p)^{j} \max \left(S_{0} u^{n-j} d^{j}-X, 0\right) .
$$

Define $C_{j}$ and $D_{j}$ as $\left(\begin{array}{c}n \\ j\end{array}\right) p^{n-j}(1-p)^{j}$ and $S_{0} u^{n-j} d^{j}$, respectively. Note that $C_{j}=C_{j-1} \times \frac{(1-p) \times(n-j+1)}{p \times j}$ and $D_{j}=D_{j-1} \times \frac{d}{u}$. Both $C_{j}$ and $D_{j}$ can be evaluated in constant time when $C_{j-1}$ and $D_{j-1}$ are known. Thus Eq. (3) can be evaluated in linear time.

Pricing double barrier options on the lattice is much more complex than pricing the vanilla options on the lattice. Traditionally, the discounted expected option payoff for the double barrier call can be solved by dynamic programming [6]. In this approach, the value at each node in the lattice is evaluated sequentially. Since there are $O\left(n^{2}\right)$ nodes in a lattice, the traditional lattice algorithm runs in $O\left(n^{2}\right)$.

\section{A Linear Time Algorithm for Double Barrier Options}

A linear-time algorithm for pricing double barrier options will be introduced in this section. First, the reflection principle and the inclusion-exclusion principle are used to derive a combinatorial formula. Second, the derived combinatorial formulas is applied to construct the pricing algorithm. Third, a proof is given to show that this algorithm runs in linear-time. 


\subsection{A Combinatorial Formula}

A combinatorial formula will be derived to count the number of price paths that reach the barrier by using the reflection principle and the inclusionexclusion principle. Consider the check graph in Fig. 2. To fit the structure of the lattice, each path in the check graph can move from vertex $(i, j)$ to vertex $(i+1, j+1)$ (the up move) or vertex $(i+1, j-1)$ (the down move). Now we consider a problem that is useful for pricing double barrier options: How may price paths starting form $(0,-a)$ will reach either barrier $H$ (horizontal line $x=0$ in the check graph) or barrier $L$ (horizontal line $x=-s$ in the check graph) before arriving at $(n,-b)$ ? Without loss of generality, we further assume that $a, b \geq 0$.

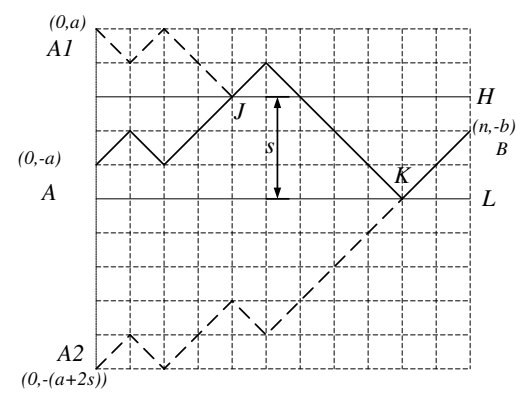

Fig. 2. Count the Number of Paths that Hit Barrier $H$ and $L$ by the Reflection Principle.

Before solving the above mentioned problem, we consider a simpler problem first: Calculate the number of the paths that move from $(0,-a)$ to $(n,-b)$ and that hit barrier $H$ before one hit of barrier $L$ by the reflection principle. One such path may hit barrier $H$ at $J$ and barrier $L$ at $K$. We can reflect the curve $\widehat{A J}$ with respect to the $H$-axis to get $\widehat{A 1 J}$. We then reflect the curve $\widehat{A 1 K}$ with respect to the $L$-axis to obtain $\widehat{A 2 K}$. By the reflection principle, the number of paths from $(0,-a)$ to $(n,-b)$ which hit the barrier $H$ at least once before hitting the barrier $L$ is equal to the number of paths that start from $(0,-(a+2 s))$ to $(n,-b)$. Assume that $x$ up moves and $y$ down moves are required to move from $(0,-(a+2 s))$ to $(n,-b)$. Thus we have $x+y=n$ and $x-y=a-b+2 s$. By solving the above two equations, $x=\frac{n+a-b+2 s}{2}$. So the answer to this problem is

$$
\left(\begin{array}{c}
n \\
\frac{n+a-b+2 s}{2}
\end{array}\right) \text { for even, non-negative } n \quad a-b .
$$

Note that a path counted by Eq. (4) may hit $L$ first before hitting $H$. The point is that among the hits, one hit of barrier $H$ must appear before one hit of barrier $L$. 
The problem of counting the number of paths that will hit either barrier $H$ or barrier $L$ before arriving at $(n,-b)$ is now within reach. First, a function $f$ is constructed to map each path to a string. This string contains the information about the barrier hitting sequence. For example, if a path $p$ hits the barrier $H$ twice before hitting the barrier $L$, then $f(p)=H H L$. Next, we define $A_{i}$ as

the set of paths whose $f$ value contains $\overbrace{H^{+} L^{+} H^{+} \ldots}^{i}$ with $i \geq 1 . L^{+}$and $H^{+}$ denote a sequence of $L \mathrm{~s}$ and $H \mathrm{~s}$, respectively. Take the path $\widehat{A B}$ in Fig. 2 as an example. $f(\widehat{A B})=H H L$, and this path belongs to both set $A_{1}$ and set $A_{2}$.

Similarly, define $B_{i}$ as the set of paths whose $f$ value contains $\overbrace{L^{+} H^{+} L^{+} \ldots}^{i}$ with $i \geq 1$. Thus the path $\widehat{A B}$ belongs to set $B_{1}$. The number of elements in set $A_{i}$ and $B_{i}$ can be calculated by using the reflection principle mentioned in last paragraph. The number of elements in each set is listed as follows:

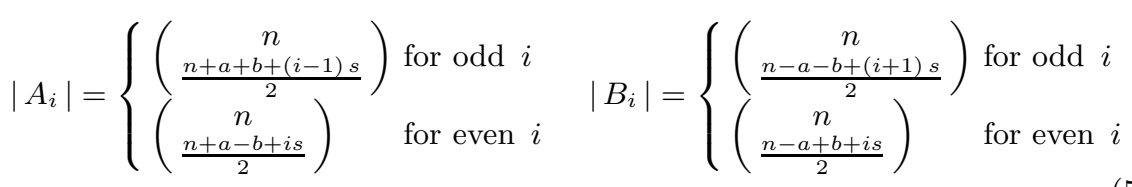

Note that each path that hits the barrier may belong to more than one set. For example, $\widehat{A B}$ in Fig. 2 belongs to $A_{1}, A_{2}$, and $B_{1}$. The inclusion-exclusion principle is applied to calculate the exact number of paths that moves from $(0,-a)$ to $(n,-b)$ and that hit either barrier $H$ or $L$ at least once as follows:

$$
\mathbf{N}(a, b, s)=\sum_{i=1}^{\left\lceil\frac{n}{s}\right\rceil}(-1)^{i+1}\left(\left|A_{i}\right| \quad\left|B_{i}\right|\right),
$$

\subsection{The Pricing Algorithm}

The construction of our algorithm can be divided into several different cases. Let us take care of degenerate cases first. If $S_{0} \leq L$, then we have $S_{\text {inf }} \leq S_{0} \leq$ $L$; that is to say, all the price paths must have reached the barrier $L$. The payoff function for the double barrier call ( see Eq. (2)) reduces to that of a vanilla call ( see Eq. (1)). Thus the option value of a double barrier call is equal to the value of a vanilla call under this case. The double barrier call can be priced by the linear-time combinatorial algorithm for pricing vanilla calls (see Eq. (3)). Similarly, if $S_{0} \geq H$, we have $S_{\text {sup }} \geq S_{0} \geq H$. The payoff of a double barrier call under this case is equal to the payoff of a vanilla call. Thus the option values of these two call options are equal and the option value can be computed by the linear-time combinatorial algorithm for pricing vanilla calls. So we only focus on the case $L<S_{0}<H$ from now on. Besides, if $X \geq H$, the payoff function for the double barrier call is again equal to that of a vanilla call. Thus the value of the double barrier call can be computed 


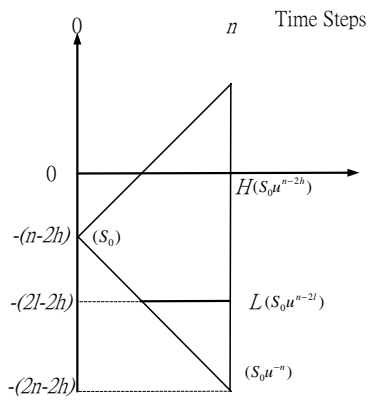

Fig. 3. Put a CRR Lattice on a Check Graph. The values in pa entheses denote the stock p ices.

by the linear-time combinatorial algorithm for pricing vanilla calls under this case . So we further focus on $X<H$ from now on.

The pricing algorithm for the non-degenerate cases is much more complex. Some terms are defined now for convenience. The term "terminal nodes" is referred to the nodes allocated at time step $n$ for convenience. We assume that

$$
V(j) \equiv p^{n-j}(1-p)^{j}\left(S_{0} u^{n-j} d^{j}-X\right),
$$

where the former term $p^{n-j}(1-p)^{j}$ denotes the probability for a price path that reaches $N(n, j)$ from the root, and the latter term $\left(S_{0} u^{n-j} d^{j}-X\right)$ denotes the payoff at node $N(n, j)$ for a price path that reaches the barrier. We further assume that

$$
V^{\prime}(k) \equiv \sum_{j=0}^{k}\left(\begin{array}{l}
n \\
j
\end{array}\right) p^{n-j}(1-p)^{j}\left(S_{0} u^{n-j} d^{j}-X\right) .
$$

Let the barrier $H=S_{0} u^{n-h} d^{h}\left(=S_{0} u^{n-2 h}\right)$, the barrier $L=S_{0} u^{n-l} d^{l}(=$ $\left.S_{0} u^{n-2 l}\right)$, and the exercise price $X=S_{0} u^{n-a} d^{a}\left(=S_{0} u^{n-2 a}\right)$. The CRR lattice can be put on a check graph as displayed in Fig. 3.

The algorithm for pricing the double barrier call is now within reach. First, a "combination table" is built for storing the value of $\left(\begin{array}{l}n \\ k\end{array}\right)$, where $0 \leq k \leq n$. Thus our pricing algorithm can evaluate $\left(\begin{array}{l}n \\ k\end{array}\right)$ in constant time by looking up this table. This design enhances the performance of our algorithm since it would cost too much time to evaluate $\left(\begin{array}{l}n \\ k\end{array}\right)$ without looking up the table. Two non-degenerate cases are discussed as follows.

Case 1. $L<X<H$ and $L<S_{0}<H$ :

The option value for the double barrier call can be divided into two parts: (1) the option value contributed by the terminal nodes between $X$ and $H$, and (2) the option value contributed by the terminal nodes at or above barrier $H$. The option value contributed by the terminal nodes between $X$ and $H$ is computed by accumulating the option value contributed by each node between $X$ and $H$. The option value contributed by a node $N(n, j)(a>j>h)$ is $e^{-r n} \mathbf{N}(n-2 h, 2 j-2 h, 2 l-2 h) V(j)$, where $\mathbf{N}(n-2 h, 2 j-2 h, 2 l-2 h)$ (defined in Eq. (6)) denotes the number of paths that reach one of the barriers before 
they reach $N(n, j)$. Thus the option value contributed by the terminal nodes between $X$ and $H$ is

$$
\left.D \equiv e^{-r n} \sum_{j=h+1}^{a} \mathbf{N}(n-2 h, 2 j-2 h, 2 l-2 h)\right) V(j) .
$$

For a price path that ends in the terminal nodes $N(n, i)$ at or above $H$ is guaranteed to reach the barrier $H$. The payoff of this price path is $\left(S_{0} u^{n-i} d^{i}-X\right)$. Note that there are $\left(\begin{array}{c}n \\ i\end{array}\right)$ paths that will reach $N(n, i)$ from $N(0,0)$. Therefore, the option value contributed by the terminal nodes at or above barrier $H$ is

$$
\sum_{j=0}^{h}\left(\begin{array}{l}
n \\
j
\end{array}\right) p^{n-j}(1-p)^{j}\left(S_{0} u^{n-j} d^{j}-X\right)=V^{\prime}(h)
$$

So we conclude that the value of the double barrier option under this case is equal to $D+V^{\prime}(h)$.

Case 2. $X \leq L$ and $L<S_{0}<H$ :

The option value for the double barrier call can be divided into three parts: (1) the option value contributed by the terminal nodes between $L$ and $H,(2)$ the option value contributed by the terminal nodes at or above barrier $H$, and (3) the option value contributed by the terminal nodes between $L$ (inclusive) and $X$.

The option value contributed by the terminal nodes between $L$ and $H$ can be computed by accumulating the option value contributed by each node between $L$ and $H$ :

$$
D^{\prime} \equiv e^{-r n} \sum_{j=h+1}^{l-1} \mathbf{N}(n-2 h, 2 j-2 h, 2 l-2 h) V(j) .
$$

The option value contributed by the terminal nodes at or above barrier $H$ is $V^{\prime}(h)$, as mentioned before.

Note that a price path that ends in a terminate node $N(n, i)$ between $L$ (inclusive) and $X$ must have reached the barrier $L$. The payoff of this price path is thus $\left(S_{0} u^{n-i} d^{i}-X\right)$. Thus the option value contributed by the terminal nodes between $L$ and $X$ is

$$
e^{-r n} \sum_{j=l}^{a}\left[\left(\begin{array}{c}
n \\
j
\end{array}\right) p^{n-j}(1-p)^{j}\left(S_{0} u^{n-j} d^{j}-X\right)\right]=V^{\prime}(a)-V^{\prime}(l-1) .
$$

So we conclude that the value of a double barrier call is $D^{\prime}+V^{\prime}(h)+V^{\prime}(a)-$ $V^{\prime}(l-1)$ under this case.

\subsection{Proof of the Linear Running Time}

We next prove that our algorithm runs in $O(n)$ time. For the degenerate cases mentioned above, the value of a double barrier call can be priced by the lineartime combinatorial algorithm for the vanilla option (see Eq. (3)). So we focus on non-degenerate cases. 
Table 1. Running-Time Comparison between the Lattice and the Combinatorial Algorithm.

\begin{tabular}{|r|c|c|c|}
\hline Period & Value & \multicolumn{2}{|c|}{ Computational Time (in seconds) } \\
\cline { 3 - 4 } & & Lattice & Combinatorics \\
\hline 1000 & 11.673462 & 02.20 & 0.0041 \\
\hline 2000 & 11.682312 & 08.74 & 0.0104 \\
\hline 3000 & 11.670069 & 19.83 & 0.0163 \\
\hline 4000 & 11.670000 & 35.04 & 0.0222 \\
\hline
\end{tabular}

The double barrier option is considered here. The initial stock price is 95 , the exercise price is 97 , the risk-free rate is $15 \%$ per annum, the volatility of the stock price is $25 \%$, and the two barriers are 120 and 80 .

Our algorithm can be divided into three parts. The first part denotes the construction of the combination table, the second part denotes the evaluation of Eq. (7), and the last part denotes the evaluation of Eq. (8) ( in case 1) or Eq. (9) ( in case 2). Our algorithm runs in linear-time if all these three parts can be solved in linear-time. First, recall that the combination table is a table that stores the value of $\left(\begin{array}{l}n \\ k\end{array}\right)$, where $0 \leq k \leq n$. The combination table could be constructed in linear-time by the fact $\left(\begin{array}{l}n \\ 0\end{array}\right)=1$ and by the recurrence equation: $\left(\begin{array}{l}n \\ k\end{array}\right)=\left(\begin{array}{l}n \\ k-1\end{array}\right) \times(n-k+1) \div k$. Next, Eq. (7) is similar to Eq. (3) and can be evaluated in linear-time by slightly modifying the algorithm that evaluates Eq. (3). So we conclude that if the last part can be evaluated in linear-time, then our algorithms runs in linear-time.

Let us consider the evaluation of Eq. (8) or Eq. (9). Note that the terms $\left|A_{i}\right|$ and $\left|B_{i}\right|$ defined in Eq. (5) can be represented in $\left(\begin{array}{c}n \\ k\end{array}\right)$ form, so $\left|A_{i}\right|+\left|B_{i}\right|$ can be evaluated in constant time by looking up the combination table. Thus $N(a, b, s)$ defined in Eq. (6) can be solved in $O\left(\left\lceil\frac{n}{s}\right\rceil\right)$ time. In both Eq. (8) and Eq. (9), $N(n-2 h, 2 j-2 h, 2 l-2 h)$ would be evaluated less than $l-h$ times. Consequently, it takes about $O\left(\frac{n}{2(l-h)}(l-h)\right) \approx O(n)$ time to evaluate Eq. (8) or Eq. (9). Thus the option value $D+V^{\prime}(h)$ ( in case 1$)$ or $D^{\prime}+V^{\prime}(h)+$ $V^{\prime}(a)-V^{\prime}(l-1)$ (in case 2$)$ can be evaluated in linear-time. So we conclude that our algorithm is a linear-time algorithm.

\section{Experimental Results}

The data in Table 1 show that the linear-time combinatorial pricing algorithm suggested in this paper is much faster than the traditional quadratic-time lattice algorithm, especially when the number of time steps tends to be large. Note that these two algorithms both produce the same pricing results. Besides, the pricing results converge to the true option value as $n \rightarrow \infty$.

The convergence behavior of the pricing results is examined in Fig. 4. The pricing results oscillate significantly when the number of time steps is small, 
and the results become more smooth as the number of time steps becomes larger. Thus an efficient pricing algorithm that can handle a large number of time steps is critical.

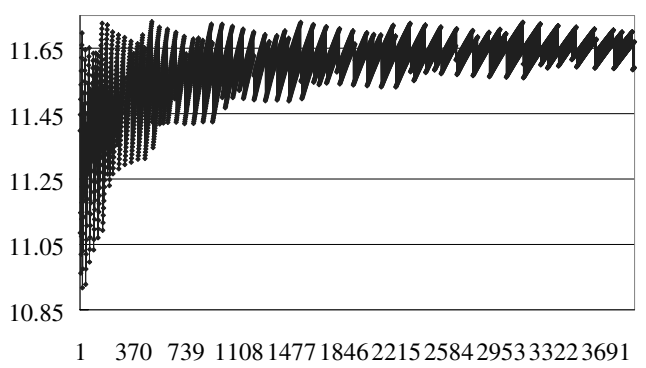

Fig. 4. Convergence of the Algorithm. The $x$-axis is the number of time steps, and the $y$-axis denotes the pricing results. Conditions are the same as in Table 1.

\section{Conclusions}

Combinatorial methods have found wide applicability in many fields. This paper extends their use in pricing double barrier options. By utilizing the reflection and the inclusion-exclusion principle, a linear-time algorithm is provided to speed up the pricing of double barrier options. Numerical experiments are given to verify our results. We expect combinatorial methods to be similarly applicable to more sophisticated path-dependent derivatives.

\section{References}

1. Bhagavatula RS, and Carr PP (1997) Valuing Double Barrier Options with Fourier Series. Manuscript, New York University, NY, USA.

2. Cox J, Ross S, Rubinstein M (1979) Option Pricing: A Simplified Approach. J. Financial Econom. 7:229-264.

3. Figlewski S, Gao B (1999) The Adaptive Mesh Model: A New Approach to Efficient Option Pricing. J. Financial Econom. 53:313-351.

4. Geman H, and Yor M (1996) Pricing and Hedging Double-Barrier Options: A Probabilistic Approach. Math. Finance 6:365-378.

5. Lyuu YD (1998) Very Fast Algorithms for Barrier Option Pricing and the Ballot Problem. J. Derivatives 5:68-79.

6. Ritchken P (1995) On Pricing Barrier Options. J. Derivatives 3:19-28.

7. Wei, JZ (1998) Valuation of Discrete Barrier Options by Interpolations. J. Derivatives, 6:51-73. 